\title{
INVESTIGATION OF FORCED CONVECTION HEAT TRANSFER FROM A HEATER MOUNTED IN A CAVITY WALL USING VARIOUS NANOFLUIDS
}

\author{
R. Kanna ${ }^{1}$, Sayed Sayeed Ahmad ${ }^{1}$, P. Venkata Reddy ${ }^{2}$, Chithirai Pon Selvan ${ }^{3}$, Tale $^{4}$, Dawid

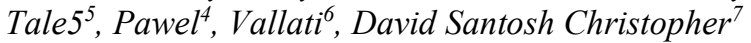 \\ ${ }^{1}$ College of Engineering and Computing, Al Ghurair University, Dubai, UAE. \\ ${ }^{2}$ Department of Mechanical Engineering, Amity University, Dubai, UAE. \\ ${ }^{3}$ School of Science and Engineering, Curtin University Dubai, UAE, \\ ${ }^{4}$ Institute of Thermal Power Engineering, Cracow University of Technology, Kraków, Poland. \\ ${ }^{5}$ Faculty of Environmental Engineering, Cracow University of Technology, 31-864 Cracow, Poland \\ ${ }^{6}$ DIAEE Sapienza University of Rome, Italy. \\ ${ }^{7}$ Department of Mechanical Engineering, Wolaita Soda University, Ethiopia,
}

\begin{abstract}
Forced convection heat transfer from heater mounted in a cavity wall is investigated to reveal the relation among nanofluid properties. The base fluid is considered as water. The present study is focused on forced convection heat transfer from square heater subject to inflow and outflow inside a square cavity. The interesting physics will be reported in connection with volume fraction, Reynolds number and nanomaterial properties. It is found that for a particular Reynolds number when nanomaterial is introduced the local heat transfer is increased. The wall attached vortex attributes a constant Nusselt number. It is also noticed that when the heater wall is subject to combination of vortex and main stream fluid results high Nusselt number than heat transfer due to wall attached vortices. Nanofluid results high Nusselt number for the same Reynolds number.
\end{abstract}

\section{INTRODUCTION}

Forced convection heat transfer from heater mounted in a cavity wall is investigated to reveal the effect of nano material properties during forced convection. The base fluid is considered as water. Saeidi and Khodadadi [1] investigated the forced convection heat transfer from a cavity for different inlet and exit ports. They found that the position of outlet port affects significantly the local Nusselt number and strength of the vortex. In [2] AhmetKoca has reported the Conjugate heat transfer in partially open square cavity with a vertical heat source. The cavity has an opening on the top with several lengths and three different positions while the other walls of cavity were assumed adiabatic. It was found that ventilation position has a significant effect on heat transfer. Poochinapan [3] has solved cavity flow for comparison of explicit and implicit scheme used for simulating nonlinear convective terms by stream function vorticity formulation. Esfe and Saedodin [4] have studied numerically the effect of nanoparticle diameter on heat transfer. They found that the nanoparticle diameter has inverse relationship with average Nusselt number. Sheikholeslamia and Vajravelu [5] have reported the effect of variable magnetic field on nanofluid heat transfer in a cavity. They reported that the main vortex moves downward however when the Lorentz force is increased and the heat transfer rate is also increased. Sheikholeslami [6] have

* Corresponding author email: prkanna@gmail.com 
conducted forced convection heat transfer in a cubical cavity using nanofluid under the presence of magnetic field. Their results depicted that forced convection reduces with enhance of Lorentz forces. Kalidasan et. al. [7] have reported natural convection from a $\mathrm{C}$ shaped cavity using nanofluid. An isothermal block was placed inside the cavity and Copper Titania hybrid nanofluid were tested. They found that When Rayleigh number increases, the strength of the primary vortex diminishes and heat transfer increased monotoniously for a particular Rayleigh number. It is found that from the open literature the there is no study reveals the forced convection using nanofluid to reveal the heat transfer from a heater mounted in cavity wall with inflow and outflow condition. The present study is focused on forced convection heat transfer from square heater mounted on a cavity wall. The interesting physics will be reported in connection with volume fraction, Reynolds number and nanomaterial properties. Use $170 \times 250$ $\mathrm{mm}$ paper size ( $\mathrm{W} \times \mathrm{H} \mathrm{mm}$ ) and adjust the margins to those shown in the Table 1 . The final printed area will be $130 \times 210$ $\mathrm{mm}$. Do not add any page numbers.

\section{Figures and tables}

Figure 1 shows the schematic of the problem considered for computation. Incompressible two-dimensional laminar flow is considered for the computation. The governing Navier-Stokes equations are solved by stream function-vorticity formulation. The transient nondimensional governing equations in the conservative form are,

Stream function equation

$$
\nabla^{2} \psi=-\omega
$$

Vorticity equation

$$
\frac{\partial \omega}{\partial t}+\frac{\partial(u \omega)}{\partial x}+\frac{\partial(v \omega)}{\partial y}=\frac{1}{\operatorname{Re}(1-\phi)^{2.5}\left[(1-\phi)+\phi \frac{\rho_{s}}{\rho_{f}}\right]} \nabla^{2}
$$

Energy equation

$$
\frac{\partial \theta}{\partial t}+\frac{\partial(u \theta)}{\partial x}+\frac{\partial(v \theta)}{\partial y}=\frac{\left(k_{n f} / k_{f}\right)}{\operatorname{RePr}\left[(1-\phi)+\phi \frac{(\rho C p)_{s}}{(\rho C P)_{f}}\right]} \nabla^{2} \theta
$$

The effective thermal conductivity of the nanofluid is approximated by Maxwell-Garnetts (MG) for fluid containing a spherical nano particle.

$$
\frac{k_{n f}}{k_{f}}=\frac{k_{s}+2 k_{f}-2 \phi\left(k_{f}-k_{s}\right)}{k_{s}+2 k_{f}+\phi\left(k_{f}-k_{s}\right)}
$$

The following boundary conditions are assumed for simulation. The cavity dimensions are assumed as $W=H=1$ and $\boldsymbol{w}=0.25$. The heater is also assumed as square of size as $\boldsymbol{w}$ and maintained at constant wall temperature. Hence the nondimensional temperature $\theta=1$ along all sides (A-B-C-D) of heater and inlet it equals to zero. The side walls of the cavity are maintained at adiabatic condition. Figure 2 shows the typical mesh used for the simulation. Table 1 shows the thermophysical properties of copper and silver nanomaterials. To ensure laminar flow the Reynolds number is restricted to maximum of 200 . The nanomaterial volume fraction is computed for $\varphi=0,5 \%$ and $10 \%$. The detailed numerical procedure for nanofluid simulation and validation can be found from Kanna et al. [8].

Figures and tables, as originals of good quality and well contrasted, are to be in their final form, ready for reproduction, pasted in the appropriate place in the text. Try to ensure that the size of the text in your figures is approximately the same size as the main text (10 point). Try to ensure that lines are no thinner than 0.25 point. 
Table 1: Thermophysical properties of base fluid and nanoparticles

\begin{tabular}{|c|c|c|c|}
\hline Property & $\begin{array}{c}\text { Pure } \\
\text { water }\end{array}$ & $\mathrm{Ag}$ & $\mathrm{Cu}$ \\
\hline$\rho$ & 997.1 & 1,0500 & 8933 \\
\hline$K$ & 0.613 & 429 & 400 \\
\hline$C_{p}$ & 4179 & 235 & 385 \\
\hline
\end{tabular}

Table 2: Average Nusselt number from heater walls

\begin{tabular}{|c|c|c|c|c|}
\hline Re & $\begin{array}{c}\text { Pure } \\
\text { water, } \\
\varphi=0\end{array}$ & $\begin{array}{c}A g, \\
\varphi= \\
5 \%\end{array}$ & \multicolumn{2}{|c|}{$C u$} \\
\hline 50 & 19.75 & - & $\begin{array}{c}\varphi= \\
5 \%\end{array}$ & $\begin{array}{c}\varphi=10 \\
\%\end{array}$ \\
\hline 100 & 22.80 & 30.15 & 26.20 & 28.90 \\
\hline 200 & 27.79 & - & - & - \\
\hline
\end{tabular}

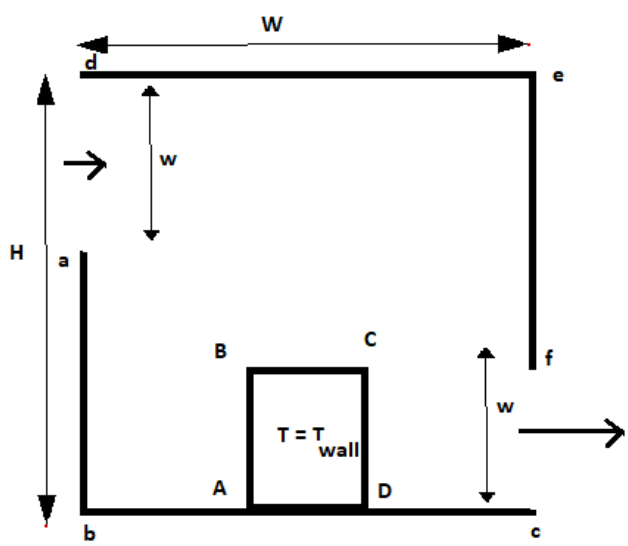

Figure 1:Schematic of the problem with boundary conditions.

\section{RESULTS DISCUSSION}

Two-dimensional laminar incompressible flow is simulated for forced convection heat transfer from a heater which is maintained at constant temperature. The fluid enters at inlet port and exit at the exit port located at the bottom of the square cavity. The side walls are maintained at adiabatic condition. Figure 3 shows the validation of flow through square cavity. The streamline contour and isothermal contor for $\mathrm{Re}=500$ is compared and found good agreement among them. Figure 4 and 5 show the effect of Reynolds number on forced convection for pure fluid. It is noticed that when Reynolds number is increased from 50 to 200 the primary vortex centre is shifted towards main stream of fluid. This is due to increase of inertia force at higher Reynolds number. Also, secondary vortices are formed at the bottom of the wall particularly cavity between heater and left side wall and top right corner of the cavity. Tertiary vorex is tend to appear at the top right corner where adverse pressure gradient is higher. It is found that when flow reaches steady state at higher Reynolds number the isotherm is denser near the heater cavity rather than main stream. This is mainly due to the strong secondary vortex appears in the vicinity of heater and side walls.

To reveal the effect of nanomaterial in the base fluid $\mathrm{Ag}$ and $\mathrm{Cu}$ are used to investigate the heat transfer. The simulations are carried out for $\mathrm{Re}=100$ and volume fraction is fixed as $5 \%$. Figure 6 shows the streamline contours for pure base fluid and figure 7 and 8 show the steamline contour for $\mathrm{Cu}$ and $\mathrm{Ag}$. It is found that at $\mathrm{Re}=100$ in addition to the primary vortex two secondary vortices are appeared in between the heater and side walls. When nano materials are introduced this two vortices size is increased and merged for high denser material. The isotherm contour for the corresponding cases discussed above are shown from figure 9 to 12 . It is noticed that the main stream fluid separates the top primary vortex from the heater. However, the bottom vorex involves intense forced convection heat transfer with the heater. To explore the effect of nanomaterial in the base fluid temperature 
distribution, temperature is plotted from y $=0.35$ along $\mathrm{x}$ direction (fig. 13). It is found that when nanomaterial is introduced the temperature is increased significantly. The local Nusselt number distribution along the walls A-B-C-D for different cases are shown from figure 14 to 16 . It is observed that the local Nusselt number from wall A-B shows a linear trend for maximum distance and decreases towards singular point. This is mainly due to the wall attached vortex at $\mathrm{Re}=100$. The top wall of heater ie. $\mathrm{B}-\mathrm{C}$ is subject to main stream of fluid and counter clockwise vortex. Hence the local $\mathrm{Nu}$ is continuously increasing upto the singular point. However, a short kink is noticed due to the conjuction of bottom wall primary vortex and main stream acting on the B-C surface. Further local $\mathrm{Nu}$ from $\mathrm{C}-\mathrm{D}$ wall shows similar to A-B wall a linear trend. The wall attached vortex attributes approximately constant $\mathrm{Nu}$ to this wall. To summarise the various cases Table 2 shows the average Nusselt number. It is evident that increasing Reynolds number will increase $\mathrm{Nu}$. When nano material is introduced in the base fluid will enhance the heat transfer. Particularly high density and low specific heat materials results high $\mathrm{Nu}$ for same Re and volume fraction. Also, it is found that $\%$ of nanomaterial increase causes increase in $\mathrm{Nu}$.

\section{CONCLUSIONS}

Two-dimensional incompressible flow over heater mounted in a square cavity is simulated subject to inflow and outflow to understand the usage of nanofluid. Results are compared with base fluid. It is found that the increase in Reynolds number will also increase in heat transfer. The wall attached vortex results less variant in local Nusselt number. The high conductivity and low specific heat nanomaterials enhance the heat transfer than the bae fluid. When parentage of volume fraction is increased proportionately the Nusselt number is also increased. The heater top wall shows higher Nusselt number than rest of the two side walls.

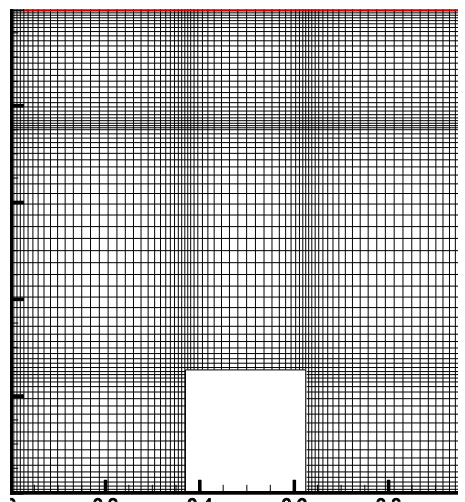

Figure 2:Computational mesh

Fig. 2 Computational mesh

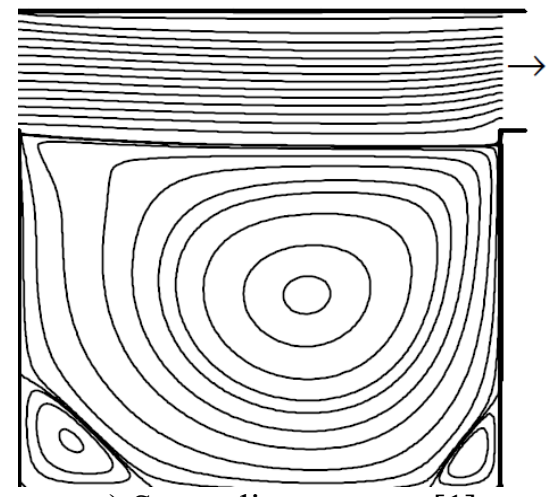

a) Streamline contour [1]

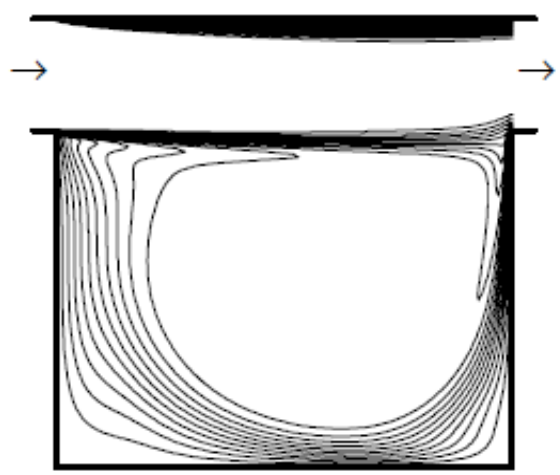


b) Isothermal contour [1]

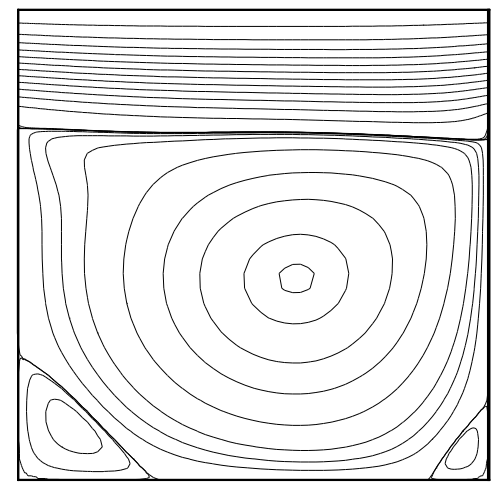

c) Streamline contour

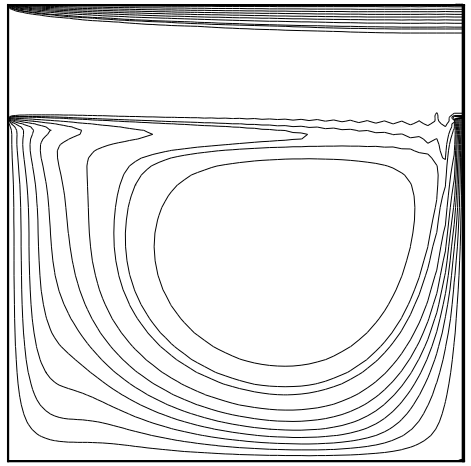

d) Isothermal contour

Figure 3 : Computational mesh

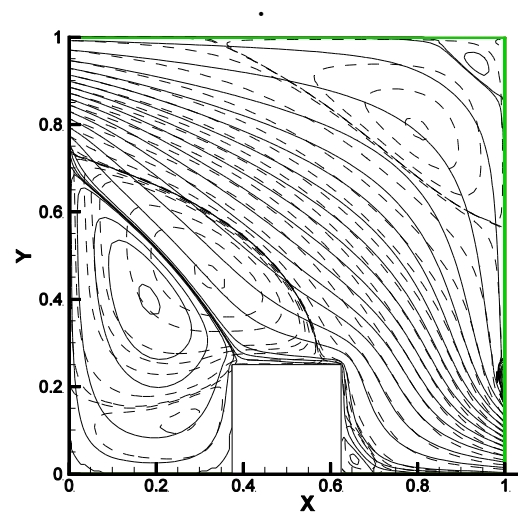

Figure 4: Streamline contour $\mathrm{Re}=$ $50(-)$ and 200(- $) . \varphi=0$

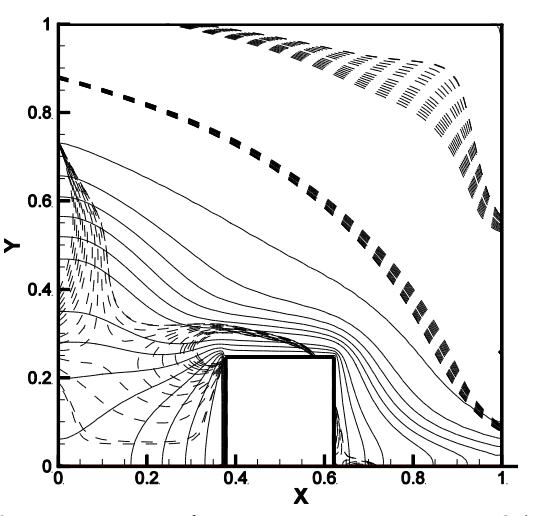

Figure 5: Isotherm contour $\mathrm{Re}=50(-$ ) and $200(--) . \varphi=0$

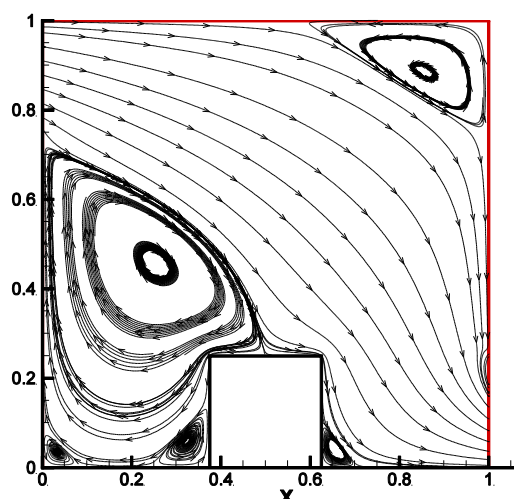

Figure 6: Streamline contour $\mathrm{Re}=$

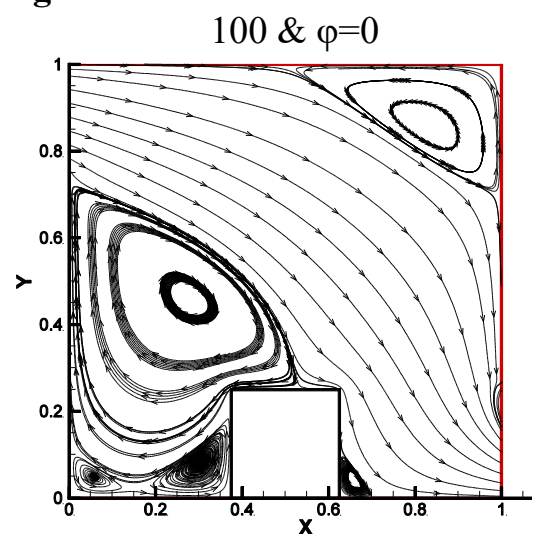

Figure 7: Streamline contour $\mathrm{Re}=$ $100, \mathrm{Cu} \& \varphi=5 \%$ 


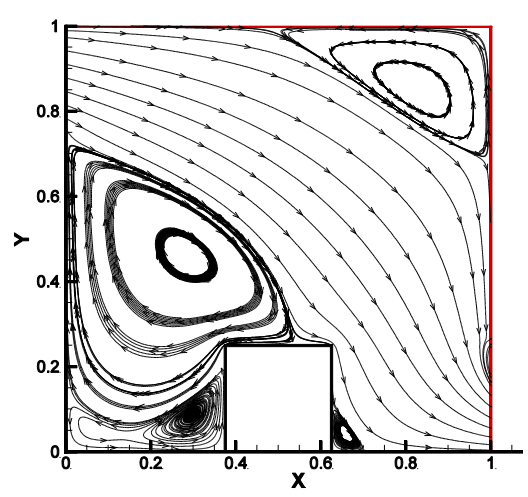

Figure 8: Streamline contour $\mathrm{Re}=$ $100, \operatorname{Ag} \& \varphi=5 \%$

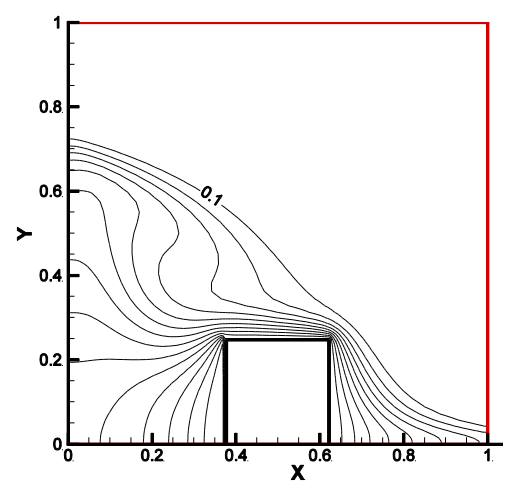

Figure 9: Isotherm contour $\mathrm{Re}=$ 100. $\varphi=0$

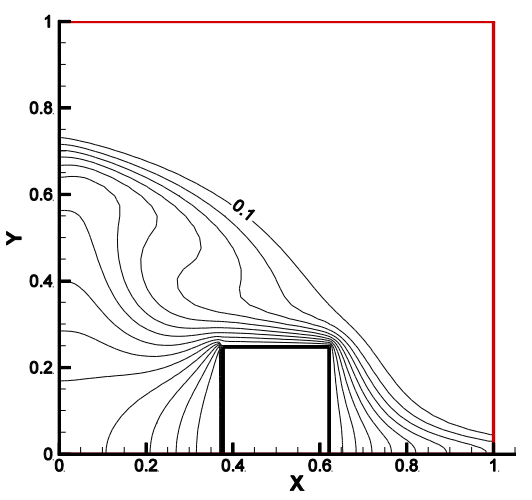

Figure 10: Isotherm contour $\mathrm{Re}=$ 100. $C u$ nanomaterial, $\varphi=5 \%$

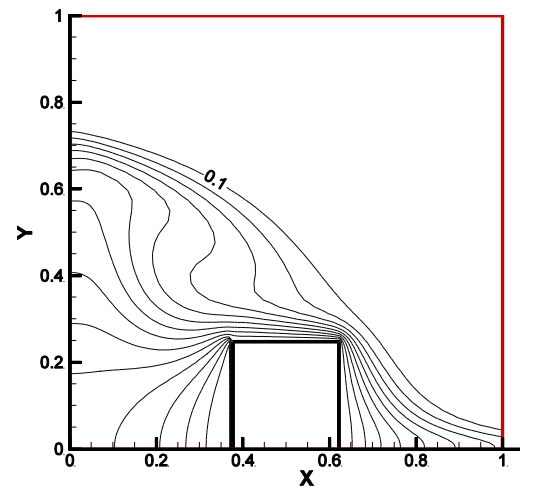

Figure 11: Isotherm contour $\mathrm{Re}=$ 100. $A g$ nanomaterial, $\varphi=5 \%$

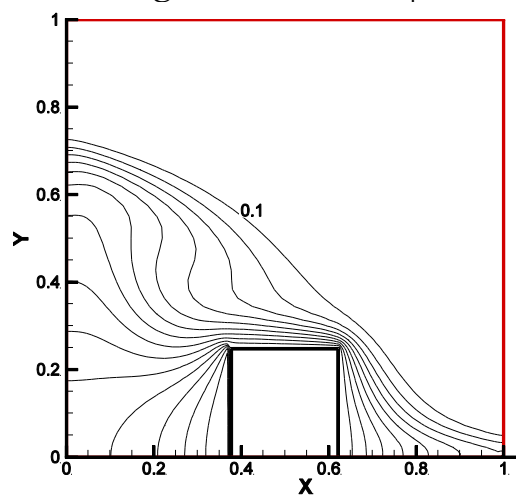

Figure 12: Isotherm contour $\mathrm{Re}=$ 100. $C u$ nanomaterial, $\varphi=10 \%$

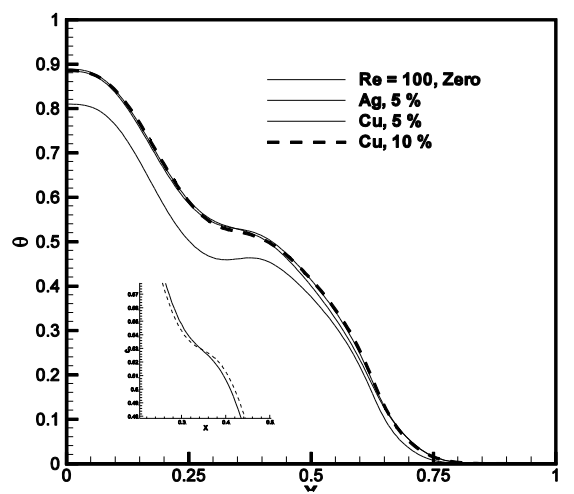

Figure 13: Isoherm at $y=0.35$ 


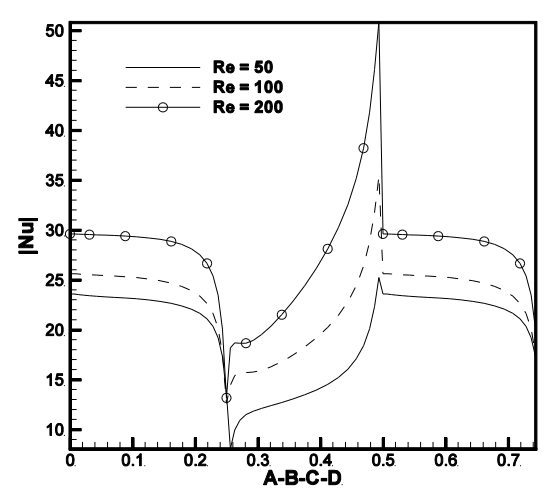

Figure 14: Local Nusselt number: pure water.

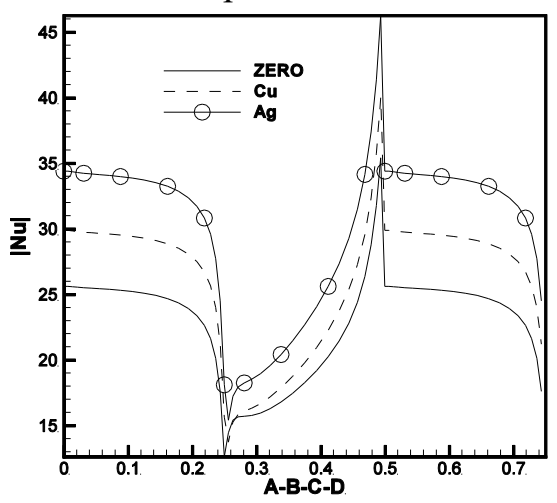

Figure 15: Local Nusselt number: Effect of material

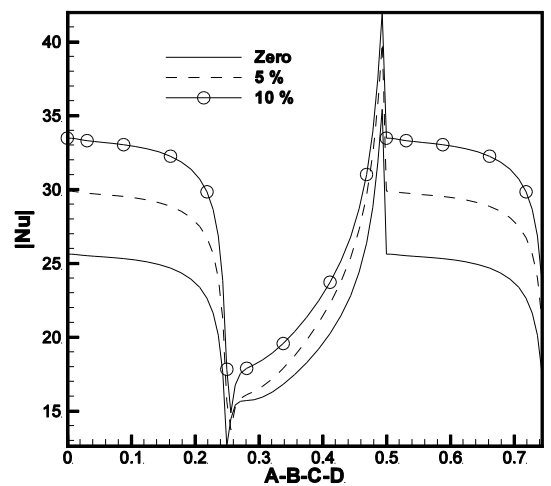

Figure 16: Local Nusselt number: Effect of volume fraction

\section{References}

1. Saeidi, S.M. and Khodadadi, J.M., Forced convection in a square cavity with inlet and outlet ports, IJHMT, 49, pp. 1896-1906 (2006).

2. AhmetKoca, Numerical analysis of conjugate heat transfer in a partially open square cavity with a vertical heat source, ICHMT, 35 pp 13851395 (2008).

3. K.Poochinapan, Numerical Implementations for 2D Lid-Driven Cavity Flow in Stream Function Formulation, Applied Mathematics, Article ID 871538, pp. 1-17 (2012).

4. Esfe, M.H., and Seyfolah Saedodin, Numerical study of a combined convection flow in a cavity filled with nanofluid considering effects of diameter of nanoparticles and cavity inclination angles, JHMTR, 5, pp. 39- 49 (2018).

5. Sheikholeslamia,M. and Vajravelu, $\mathrm{K}$,Nanofluid flow and heat transfer in a cavity with variable magnetic field, AMC, 28, pp. 272-282 (2017).

6. Sheikholeslami and Hayat and Alsaedi,Numerical simulation of nanofluid forced convection heat transfer improvement in existence of magnetic field using lattice Boltzman method, IJHMT, 108, pp.1870-1883 (2017).

7. Kalidasan, K, Velkennedy, $\mathrm{R}$ and Kanna.P.R, Laminar natural convection of Copper - Titania/Water hybrid nanofluid in an open ended $\mathrm{C}$ - shaped enclosure with an isothermal block, JML, 246, pp. 251-258 (2017).

8. Kanna. P.R., Jan Taler, Anbumalar., V, Santhosh Kumar, A.V., Allwyn Pushparaj and Christopher.D.,V.,Numerical Investigation of Conjugate Heat 
Transfer from Sudden Expansion using nano fluid, Numerical Heat
Transfer Part A. 67, pp. 75-99 (2015). 